Einführung in das Schwerpunktthema

\title{
Governancestrukturen für Nachhaltigkeit
}

S Von Ulrich Petschow nance auch in der deutschen Debatte auf der Agenda (1). Gleichwohl existiert keine einheitliche Interpretation des Begriffes, vielmehr wird dieser je nach Kontext unterschiedlich interpretiert (2). Seinen Reiz erhält der Begriff vor allen Dingen durch die Offenheit, indem nicht allein staatliche Institutionen einen wesentlichen Beitrag zur Steuerung leisten, sowie durch die allenthalben zu beobachtenden veränderten Steuerungsstrukturen - Stichwort: Partizipation im weitesten Sinne. Diese sind ein Ausdruck der veränderten Rolle des Staates sowohl nach innen, aufgrund des Komplexitätsproblems moderner Gesellschaften, als auch nach außen durch die Globalisierung.

Die von Zürn aufgestellte Triade: ,governance by government", ,governance with government" und "governance without government" verweist darauf, dass der Staat nur ein Akteur in entsprechenden Arrangements ist (3). Im Bereich der Integrierten Produktpolitik, die insbesondere durch den Ansatz der geteilten Verantwortung, also governance with government gekennzeichnet ist, ist zu fragen, wie die kontinuierlichen Verbesserungsprozesse ohne Hierarchie zu Stande kommen können (4). Im Rahmen dessen was als Corporate Governance bezeichnet wird, geht es zu aller erst um das Verhältnis zwischen Kapitalgebern und Unternehmensmanagement, also vordergründig um governance without government. Gleichwohl sind hier auch die rechtlichen Aspekte mit einzubeziehen.

Die Karriere des Begriffs beruht auch auf einem vielfach empfundenen Unbehagen mit den vorhandenen Strukturen und Entscheidungsverfahren, die oft als nicht mehr adäquat empfunden werden. Die vorbereitenden Arbeiten zu einem demnächst erscheinenden Weißbuch zu Governance in der Europäischen Union (vgl. dazu den Beitrag von Paterson) machen unter anderem deutlich, dass auf dem Weg zu neuen Formen der Governance auch die institutionellen Arrangements verändert und angepasst werden müssen. Governance ist nicht per se eine Problemlösung, sondern eher eine ergebnisorientierte Fragestel- lung, welche Steuerungsmodi erforderlich sein könnten um ein bestimmtes Ziel zu erreichen. Mit veränderten Steuerungsformen sind aber zugleich Verteilungs- und Machtfragen verbunden.

Die Beiträge dieses Schwerpunkts befassen sich mit Governance auf unterschiedlichen Ebenen. Gemeinsam ist allen Beiträgen, dass sie Governance explizit in einen Zusammenhang mit für den Nachhaltigkeitsdiskurs zentralen Fragen und Zielen stellen (5). Im Zentrum steht die Berücksichtigung bzw. Einbeziehung neuer Akteure und ihr möglicher Beitrag zu einer governance of sustainability.

\section{- Die Beiträge im Überblick}

John Paterson gibt einen Einblick in die Diskussionen in der Vorbereitung des EU-Weißbuchs zu Governance. Er verweist auf Grenzen des demokratischen Legitimationsprozesses, der den modernen Nationalstaat geprägt hat. Das demokratische Defizit, das der EU zugeschrieben wird, ist daher nicht allein durch Kompetenzstärkung des Europaparlaments zu überwinden. Zentrale zu berücksichtigende Elemente sind: (i) Überwindung des begrenzten Problemverständnisses, (ii) Stärkung partizipativer Elemente, (iii) Ungleichheit der Akteure, (iv) kontinuierliche Evaluation und Revision von Politiken und (v) gegenseitiges Lernen.

Ulrich Petschow und Jens Clausen fragen, ausgehend vom Problem des unbeschränkten Zugangs (open access) zu den globalen Gemeinschaftsgütern (global commons), nach möglichen Steuerungsstrukturen auf internationaler Ebene. Dabei wird insbesondere auf die Legitimität und die Verantwortlichkeit der unterschiedlichen Akteure sowie auf Transparenz und Informationsverfügbarkeit abgehoben.

Charlotte Streck stellt dann das Modell der Umweltpolitik in globalen Netzen vor, beispielhaft aufgezeigt an der Globalen Umweltfazilität. Sie betont die Fragmentierung und die begrenzten Erfolge der zwischenstaatlichen Zusammenarbeit. Sie plädiert für die Stärkung flexibler trisektoraler Netzwerke gerade in bislang blockierten Bereichen, um neue Lösungsansätze auszuloten. Als mögliche institutionelle Struktur bzw. Plattform hierfür schlägt sie das UN Umweltprogramm UNEP vor.
Volker Schneider und Karsten Ronit untersuchen am Beispiel Farbstoffgifte den Beitrag, den internationale Unternehmerorganisationen zur Bereitstellung öffentlicher Güter leisten (können), und entdecken hier wenig wahrgenommene Potenziale. Selbstregulierungsansätze benötigen jedoch eine „komplementäre Hintergrundkontrolle“ unter anderem durch öffentliche Institutionen, wissenschaftliche Gesellschaften oder auch NGOs, um durchsetzungsfähig zu sein.

Der Beitrag von Jan Aart Scholte untersucht die Rolle der Zivilgesellschaft im globalen Finanzsystem. Er verweist darauf, dass diese vorrangig die Folgen der finanziellen Arrangements thematisiert, wie etwa der Strukturanpassungsprogramme, und sich erst in jüngerer Zeit sich mit Themen wie Tobin-Steuer oder ethisches Investment befasst. Zugleich versucht er, Vor- und Nachteile dieses Engagements abzuwägen.

Guido Nischwitz und Reimar Molitor beschäftigen sich schließlich vor dem Hintergrund der Veränderung der Bedeutung der Region und des Konzepts der nachhaltigen Entwicklung mit dem noch wenig konkretisierten Konzept der Regional Governance. Sie interpretieren es als ein Instrument, das für die Verknüpfung der drei Steuerungsformen Markt, Hierarchie und Netzwerke steht, und betonen einen erheblichen Forschungsund Handlungsbedarf. Ein solcher lässt sich zweifellos für das gesamte hier behandelte Themengebiet konstatieren.

\section{Anmerkungen}

(1) Vgl. Stiftung Entwicklung und Frieden (Hg.): Nachbarn in Einer Welt. Der Bericht der Commission on Global Governance, Bonn 1995.

(2) Vgl. Mürle, H.: Global Governance. Literaturbericht und Forschungsfragen. INEF Report, Heft 32. Duisburg 1998.

(3) Zürn, M.: Regieren jenseits des Nationalstaates. Globalisierung und Denationalisierung als Chance, Frankfurt a.M. 1998.

(4) Vgl. dazu auch den Schwerpunkt Integrierte Produktpolitik in Ökologisches Wirtschaften Nr. 6/2000.

(5) Einen guten Überblick über den Zusammenhang von Global Governance und Umweltpolitik gibt die Ausgabe 2/1998 von Ökologisches Wirtschaften.

\section{Der Autor}

Ulrich Petschow ist Leiter des Forschungsfelds Umweltökonomie und -politik am Institut für ökologische Wirtschaftsforschung.

Kontakt: IÖW, Potsdamer Str. 105, 10785 Berlin. Tel. 030/ 884594-23, Fax 030/ 8825439, E-mail: ulrich.petschow@ioew.de 
(c) 20I0 Authors; licensee IÖW and oekom verlag. This is an article distributed under the terms of the Creative Commons Attribution Non-Commercial No Derivates License (http://creativecommons.org/licenses/by-nc-nd/3.o/), which permits unrestricted use, distribution, and reproduction in any medium, provided the original work is properly cited. 